\title{
New synthesis of substituted 6-methylbenzo[b]furo-, -thieno-, and -seleno[2,3-c]quinolines, and heterocyclic analogues
}

\author{
S. Deprets and G. Kirsch \\ Laboratoire d'Ingéniérie Moléculaire et de Biochimie Pharmacologique \\ Université de Metz, Ile du Saulcy, F-57045 METZ Cedex 1 \\ E-mail: kirsch@bridoux.sciences.univ-metz.fr
}

\begin{abstract}
Substituted 6-methylbenzo[b]furo, -thieno-, and -seleno[2,3-c]quinolines were synthetized either by thermal electrocyclisation of the 2-ethanonoximes of 3-phenyl- and 3-hetaryl-substituted benzo[b]furans, -thiophenes, and selenophene, or by treatment of the corresponding $O-2,4-$ dinitrophenyl oximes with sodium hydride.
\end{abstract}

Keywords: Coupling, triflates, benzo[b]hetarylquinolines,electrocyclisation ,oximes

\section{Introduction}

We present here the synthesis of 6-methylbenzo[b]furo-, thieno- and seleno[2, 3-c]quinolines 1 and their analogues derived by replacing the benzene ring of the quinoline moiety either by thiophene 2 or furan 3.
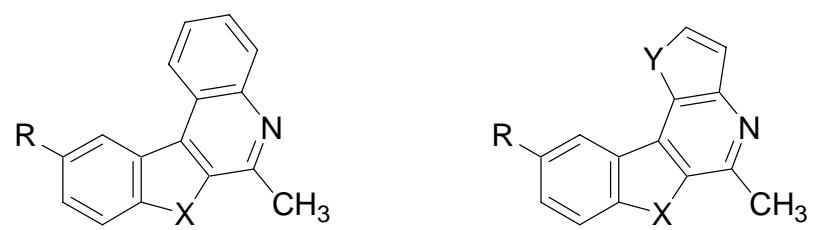

$$
\begin{aligned}
& \text { 1a }(R=B r, X=O) \\
& \text { 1b }(R=C l, X=O) \\
& \text { 1c }(R=H, X=S) \\
& \text { 1d }(R=H, X=S e)
\end{aligned}
$$

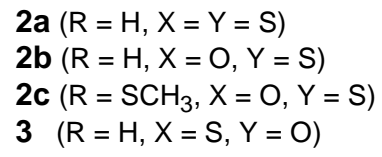

6-Methylbenzo[b]furo[2,3-c]quinoline 1 has previously been obtained from 2-amino-2'hydroxybenzophenone. ${ }^{1}$ However, this method can not lead to 6-methylbenzo[b]thieno- and seleno[2,3-c] quinolines $\mathbf{1}$ ( $\mathrm{X}=\mathrm{S}$ or $\mathrm{Se}$ ) or to the heterocyclic analogues $\mathbf{2}$ and $\mathbf{3}$. Hence, we have 
developed a novel and general method for the preparation of compounds 1, $\mathbf{2}$ and $\mathbf{3}$ starting from readily available 2-acetyl-3-bromo or 2-acetyl-3-(trifluoromethanesulfonyloxy)benzo[b]furan, benzo[b]thiophene and -benzo[b]selenophene.

\section{Results and Discussion}

A key step of this strategy is the preparation of 3-hetaryl-substituted 2-acetylbenzo[b]furan, -thiophene and -selenophene derivatives 8 by palladium-catalysed cross-coupling between 3-bromo-2-acetylbenzo[b]thiophene $\mathbf{4 a}$, -selenophene $\mathbf{4 b}$ or diversely substituted 3-trifluoromethanesulfonyloxy)benzo[b]furans $\mathbf{5 a - c}$, -thiophene $\mathbf{5 d}$ and various organostannanes $\mathbf{6 a - c}$ or organoboronic acids $\mathbf{7 a - c}$. Compounds $\mathbf{4 a - b}$ were obtained from 3-oxobenzo[b]thiophene or selenophene, and the 3-trifluoromethanesulfonyloxy derivatives 5a-d were prepared from the corresponding hydroxy derivatives as described earlier., ${ }^{2,6}$

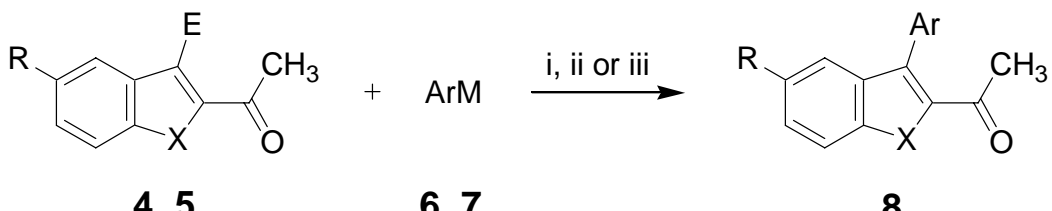
4,5
6, 7
8

Scheme 1. i: $\operatorname{ArB}(\mathrm{OH})_{2}, 2 \mathrm{~N} \mathrm{Na} \mathrm{CO}_{3}, \operatorname{Pd}\left(\mathrm{PPh}_{3}\right)_{4}, \mathrm{DME}$ (for $\mathrm{E}=\mathrm{Br}$ ); ii: $\operatorname{ArB}(\mathrm{OH})_{2}$, CuI, $2 \mathrm{~N}$ $\mathrm{Na}_{2} \mathrm{CO}_{3}, \mathrm{Pd}\left(\mathrm{PPh}_{3}\right)_{4}$, toluene (E = OTf); iii: $\mathrm{ArSnBu}_{3}, \mathrm{CuBr}_{2}, \mathrm{Pd}\left(\mathrm{PPh}_{3}\right)_{4}$, dioxane (for $\mathrm{E}=\mathrm{Br}$ or OTf). For $\mathrm{R}, \mathrm{X}$, and Ar see Table 1.

Table 1. Palladium cross-coupling reactions

\begin{tabular}{ccccccc}
\hline Starting compound & $\mathrm{X}$ & $\mathrm{R}$ & $\mathrm{E}$ & $\mathrm{ArM}^{*}$ & Product & Yield [\%] \\
\hline $\mathbf{5 a}$ & $\mathrm{O}$ & $\mathrm{Br}$ & $\mathrm{OTf}$ & $\mathbf{6 a}$ & $\mathbf{8 a}$ & 52 \\
$\mathbf{5 a}$ & $\mathrm{O}$ & $\mathrm{Br}$ & $\mathrm{OTf}$ & $\mathbf{7 a}$ & $\mathbf{8 a}$ & 47 \\
$\mathbf{5 b}$ & $\mathrm{O}$ & $\mathrm{Cl}$ & $\mathrm{OTf}$ & $\mathbf{6 a}$ & $\mathbf{8 b}$ & 87 \\
$\mathbf{5 b}$ & $\mathrm{O}$ & $\mathrm{Cl}$ & $\mathrm{OTf}$ & $\mathbf{7 a}$ & $\mathbf{8 b}$ & 78 \\
$\mathbf{5 b}$ & $\mathrm{O}$ & $\mathrm{H}$ & $\mathrm{OTf}$ & $\mathbf{6 b}$ & $\mathbf{8 c}$ & 89 \\
$\mathbf{5 b}$ & $\mathrm{O}$ & $\mathrm{H}$ & $\mathrm{OTf}$ & $\mathbf{7 b}$ & $\mathbf{8 c}$ & 71 \\
$\mathbf{5 c}$ & $\mathrm{O}$ & $\mathrm{SCH}$ & $\mathrm{OTf}$ & $\mathbf{6 b}$ & $\mathbf{8 d}$ & 94 \\
$\mathbf{4 a}$ & $\mathrm{S}$ & $\mathrm{H}$ & $\mathrm{Br}$ & $\mathbf{6 a}$ & $\mathbf{8 e}$ & 82 \\
$\mathbf{4 a}$ & $\mathrm{S}$ & $\mathrm{H}$ & $\mathrm{Br}$ & $\mathbf{7 a}$ & $\mathbf{8 e}$ & 76 \\
$\mathbf{5 d}$ & $\mathrm{S}$ & $\mathrm{H}$ & $\mathrm{OTf}$ & $\mathbf{7 a}$ & $\mathbf{8 e}$ & 69 \\
$\mathbf{4 a}$ & $\mathrm{S}$ & $\mathrm{H}$ & $\mathrm{Br}$ & $\mathbf{6 c}$ & $\mathbf{8 f}$ & 82 \\
$\mathbf{5 d}$ & $\mathrm{S}$ & $\mathrm{H}$ & $\mathrm{OTf}$ & $\mathbf{6 c}$ & $\mathbf{8 f}$ & 77 \\
$\mathbf{5 d}$ & $\mathrm{S}$ & $\mathrm{H}$ & $\mathrm{OTf}$ & $\mathbf{6 b}$ & $\mathbf{8 g}$ & 66 \\
\hline
\end{tabular}


Table 1. Continued

\begin{tabular}{ccccccc}
\hline $\mathbf{4 b}$ & $\mathrm{Se}$ & $\mathrm{H}$ & $\mathrm{Br}$ & $\mathbf{6 a}$ & $\mathbf{8 h}$ & 87 \\
$\mathbf{4 b}$ & $\mathrm{Se}$ & $\mathrm{H}$ & $\mathrm{Br}$ & $\mathbf{7 a}$ & $\mathbf{8 h}$ & 94 \\
\hline
\end{tabular}

* $\mathbf{6 a}=\mathrm{PhSnBu}_{3}, \mathbf{6 b}=$ 2-thienyl $\mathrm{SnBu}_{3}, \mathbf{6 c}=$ 2-furylSnBu $3 ;$ 7a $=\mathrm{PhB}(\mathrm{OH})_{2}, \mathbf{7 b}=$ 2-thienyl $\mathrm{B}(\mathrm{OH})_{2}, 7 \mathrm{c}=2$-furylB $(\mathrm{OH})_{2}$

Coupling between the substituted 2-acetyl-3-bromo or 2-acetyl-3-trifluoromethanesulfonyloxy)benzo[b]furans, -thiophenes and -selenophene $\mathbf{4}$ and $\mathbf{5}$, respectively, and boronic acids 7 using a modification of Suzuki's procedure ${ }^{3 \mathrm{~b}, \mathrm{c}}$ (Scheme 1) afforded the biaryl derivatives $\mathbf{8 a}-\mathbf{h}$ in moderate to good yields (Table1). Similar yields of coupling products $\mathbf{8}$ were obtained by applying Stille's conditions using organostannanes 6. The Stille reaction was carried out in refluxing dioxane in the presence of $\mathrm{Pd}^{0}$ and $\mathrm{CuBr}_{2}$ as co-catalysts ${ }^{4}$ (Scheme 1, Table1).

The ketoximes 9a-h were prepared in almost quantitative yields by refluxing the corresponding ketones $\mathbf{8 a}-\mathbf{h}$ in ethanol with hydroxylamine hydrochloride and sodium acetate.

Cyclisation of oximes 9 turned out to be the critical step in the synthesis of the tetracyclic compounds 1, 2 and 3. Thermal electrocyclisation of oximes 9 in diphenylether at $190{ }^{\circ} \mathrm{C}$ afforded the 6-methylbenzo[b]furo-, -thieno- or -seleno[2,3-c]quinolines $\mathbf{1}$ and their analogues 2 and 3 in yields lower than $20 \%$.

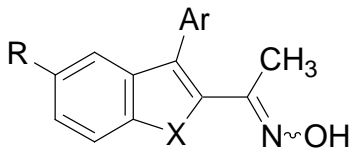

9

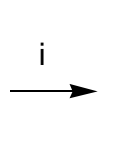

r

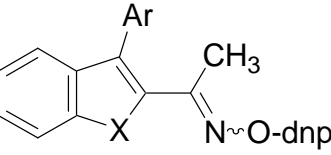

10

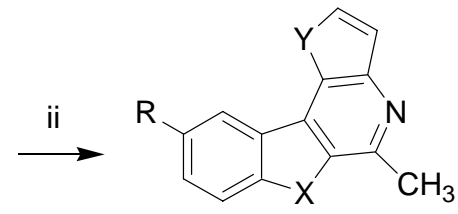

$1,2,3$

Scheme 2. i: $\mathrm{NaH} 60 \%$, THF, 1-chloro-2,4-dinitrobenzene ( $\mathrm{dnp}=2,4-\mathrm{O}_{2} \mathrm{NC}_{6} \mathrm{H}_{3}$ ); ii: $\mathrm{NaH} 60 \%$, dioxane. For R, X, and Ar see Table 2.

In order to improve the yields, the cyclisation was accomplished by activating oximes $\mathbf{9}$ as O-2,4-dinitrophenyl derivatives as it has been shown by Narasaka and al. ${ }^{5}$ The activated compounds 10a-h, prepared by reacting the sodium salt of the oximes 9 with 1-chloro-2,4dinitrobenzene, were cyclised using sodium hydride in dioxane (Table 2, Scheme 2). When compound 10b $(\mathrm{R}=\mathrm{Cl})$ was subjected to these conditions, cyclization occurred already at room temperature. Longer reaction times were required to cyclise compounds $\mathbf{1 0}$ which are substituted by a furan or a thiophene ring in position 3 (Table 2). 
Table 2. Cyclisation of $O$-2, 4-dinitrophenyloximes 10

\begin{tabular}{cllcccc}
\hline Product & $\mathrm{R}$ & $\mathrm{X}$ & $\mathrm{Ar}$ & $\mathrm{Y}$ & Reaction time (h) & Yield (\%) \\
\hline 1a & $\mathrm{Br}$ & $\mathrm{O}$ & phenyl & $\mathrm{CH}=\mathrm{CH}$ & 4 & 96 \\
1b & $\mathrm{Cl}$ & $\mathrm{O}$ & phenyl & $\mathrm{CH}=\mathrm{CH}$ & 3 at r.t. & 97 \\
$\mathbf{1 c}$ & $\mathrm{H}$ & $\mathrm{Se}$ & phenyl & $\mathrm{CH}=\mathrm{CH}$ & 6 & 84 \\
$\mathbf{1 d}$ & $\mathrm{H}$ & $\mathrm{S}$ & phenyl & $\mathrm{CH}=\mathrm{CH}$ & 4 & 64 \\
2a & $\mathrm{H}$ & $\mathrm{S}$ & thienyl & $\mathrm{S}$ & 22 & 56 \\
2b & $\mathrm{H}$ & $\mathrm{O}$ & thienyl & $\mathrm{S}$ & 24 & 89 \\
2c & $\mathrm{SCH}_{3}$ & $\mathrm{O}$ & thienyl & $\mathrm{S}$ & 25 & 63 \\
$\mathbf{3}$ & $\mathrm{H}$ & $\mathrm{S}$ & furyl & $\mathrm{O}$ & 30 & 82 \\
\hline
\end{tabular}

\section{Experimental Section}

General Procedures. Melting points were determined on a Stuart Scientific melting point appratus MP3. ${ }^{1} \mathrm{H}$ NMR spectra were recorded on an AC Bruker $250 \mathrm{MHz}$ instrument. Infrared spectra (IR) were measured on a Perkin-Elmer 881 spectrometer. Compounds 4a-b were prepared as described. ${ }^{6} \mathrm{CH}_{3} \mathrm{CN}$ was distilled over potassium carbonate; DME was distilled over lithium aluminium hydride. Silica gel (chromagel 70-200 $\mu \mathrm{m}$ ) was used for column chromatography.

\section{General procedures for the Suzuki cross-coupling reactions}

Procedure 1. Bromo derivatives 4a-b (1 mmol) were dissolved in DME $(50 \mathrm{~mL})$ and the resultant solution was purged with nitrogen. $\mathrm{Pd}\left(\mathrm{PPh}_{3}\right)_{4}\left(1.1 \mathrm{mg}, 10^{-3} \mathrm{mmol}, 3 \mathrm{~mol} \%\right)$ was added; the mixture was stirred for 15 minutes, treated with $2 \mathrm{~N}$ aqueous sodium carbonate $(2 \mathrm{~mL})$ and the solution became cloudy. The boronic acids ${ }^{8} \mathbf{7 a}-\mathbf{c}(1.1 \mathrm{mmol}$.) were added as a solid. The solution was heated under reflux until $\mathbf{4 a} \mathbf{a}-\mathbf{b}$ was consumed (monitored by TLC). The solvent was removed in vacuum, and the residue was partitioned between diethyl ether $(20 \mathrm{~mL})$ and water $(10 \mathrm{~mL})$. The organic layer was separated and washed with brine, dried over $\mathrm{Na}_{2} \mathrm{SO}_{4}$ and concentrated in vacuum. The residue was purified by column chromatography using $\mathrm{CH}_{2} \mathrm{Cl}_{2}$ as eluent to give $\mathbf{8 e}-\mathbf{h}$.

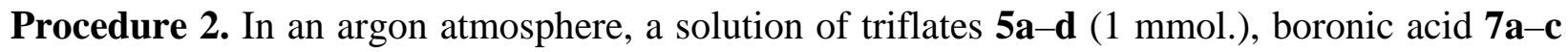
(3 mmol.), copper(I) iodide (209 mg, $1.1 \mathrm{mmol}$.), $2 \mathrm{~N}$ sodium carbonate (742 mg, $7 \mathrm{mmol}$.) and $\mathrm{Pd}\left(\mathrm{PPh}_{3}\right)_{4}$ (34 mg, 3.10 $\left.{ }^{-3} \mathrm{mmol}, 4 \mathrm{~mol} \%\right)$ in dry toluene $(10 \mathrm{~mL})$ was heated under reflux until 5a-d has disappeared (TLC). The reaction mixture was then cooled to room temperature, and water and ethyl acetate were added. The two layers were partitioned, and the organic phase was washed with brine, dried over $\mathrm{Na}_{2} \mathrm{SO}_{4}$ and concentrated under vacuum. The residue was purified by column chromatography using $\mathrm{CH}_{2} \mathrm{Cl}_{2}$ as eluent to give $\mathbf{8}$. 


\section{General procedure for the Stille cross coupling reactions}

A solution of stannane $\mathbf{6 a}-\mathbf{c}(1.04 \mathrm{mmol})$, bromo or triflate derivative $\mathbf{4 a , b}$ or $\mathbf{5 a - d}(1 \mathrm{mmol})$, respectively, $\mathrm{LiCl}$ (105 mg, $3 \mathrm{mmol}$ ) and $\mathrm{Pd}\left(\mathrm{PPh}_{3}\right)_{4}$ (34 mg, 3.10 ${ }^{-3} \mathrm{mmol}, 4 \mathrm{~mol} \%$ ) in dioxane (4 $\mathrm{mL}$ ) were refluxed in an argon atmosphere until disappearance of the triflate or the bromide (TLC). The reaction mixture was then cooled to room temperature, and water (40 mL) and ethyl acetate $(25 \mathrm{~mL})$ were added. The two layers were partitioned, the organic phase was separated, washed with brine, dried over $\mathrm{Na}_{2} \mathrm{SO}_{4}$ and concentrated under vacuum. The residue was purified by column chromatography using $\mathrm{CH}_{2} \mathrm{Cl}_{2}$ as eluent to give $\mathbf{8}$.

1-(5-Bromo-3-phenylbenzofuran-2-yl)ethanone (8a). Colorless crystals, mp $99{ }^{\circ} \mathrm{C}$. IR (KBr): 1686 (CO) $\mathrm{cm}^{-1}$; ${ }^{1} \mathrm{H}$ NMR $\left(\mathrm{CDCl}_{3}\right): \delta 2.49$ (s, 3H, $\left.\mathrm{CH}_{3}\right) 7.48$ (d, J= $\left.7.65 \mathrm{~Hz}, 1 \mathrm{H}, \mathrm{ArH}\right), 7.52$ (m, 5H, ArH), 7.60 (dd, $J=1.67,8.67 \mathrm{~Hz}, 1 \mathrm{H}, \mathrm{ArH}), 7.70$ (d, $J=1.68 \mathrm{~Hz}, 1 \mathrm{H}$, ArH). Anal. Cald. for $\mathrm{C}_{16} \mathrm{H}_{10} \mathrm{BrO}_{2}$ (315.16): C, 60.97; H, 3.52. Found: C, 60.91, H, 3.45.

1-(5-Chloro-3-phenylbenzofuran-2-yl)ethanone (8b). Colorless crystals, mp $87{ }^{\circ} \mathrm{C}$. IR (KBr):1679 (CO) $\mathrm{cm}^{-1} ;{ }^{1} \mathrm{H}$ NMR $\left(\mathrm{CDCl}_{3}\right): \delta 2.49$ (s, 3H, $\left.\mathrm{CH}_{3}\right), 7.48(\mathrm{~m}, 1 \mathrm{H}, \mathrm{ArH}), 7.57$ (m, 7H, ArH). Anal. Cald. for $\mathrm{C}_{16} \mathrm{H}_{10} \mathrm{ClO}_{2}$ (270.70): C, 70.98; H, 3.69. Found: C, 71.08; H, 3.76.

1-[(3-Thiophen-2-yl)benzofuran-2-yl]ethanone (8c). Colorless crystals, mp $82{ }^{\circ} \mathrm{C}$. IR (KBr): $1673(\mathrm{CO}) \mathrm{cm}^{-1}$; ${ }^{1} \mathrm{H}$ NMR $\left(\mathrm{CDCl}_{3}\right): \delta 2.62$ (s, 3H, $\left.\mathrm{CH}_{3}\right), 7.21$ (dd, $J=2.8,4.2 \mathrm{~Hz}, 1 \mathrm{H}, \operatorname{ArH}$ ), 7.36 (m, 1H, ArH), 7.55 (m, 3H, ArH), 7.72 (d, $J=2.8 \mathrm{~Hz} 1 \mathrm{H}, \mathrm{ArH}), 7.93$ (d, $J=7.98 \mathrm{~Hz}, 1 \mathrm{H}$, $\mathrm{ArH}) ;{ }^{13} \mathrm{C} \mathrm{NMR}\left(\mathrm{CDCl}_{3}\right): \delta 189.7$ (CO), 153.9, 146.9, 130.9, 127.9, $120.6\left(\mathrm{C}_{\mathrm{q}}\right), 130.0,128.4$, 127.2, 124.0, 123.0, $112.2(\mathrm{CH}), 28.2\left(\mathrm{CH}_{3}\right)$. Anal. Cald. for $\mathrm{C}_{14} \mathrm{H}_{10} \mathrm{O}_{2} \mathrm{~S}$ (242.22): C, 69.41; H, 4.16. Found: C, 69.54; H, 4.25.

1-[(5-Methylsulfanyl-3-thiophen-2-yl)benzofuran-2-yl]ethanone (8d). Colorless crystals, mp $61{ }^{\circ} \mathrm{C}$. IR (KBr): 1672 (CO) $\mathrm{cm}^{-1} ;{ }^{1} \mathrm{H}$ NMR $\left(\mathrm{CDCl}_{3}\right): \delta 2.54$ (s, 3H, $\left.\mathrm{CH}_{3}\right), 2.6\left(\mathrm{~s}, 3 \mathrm{H}, \mathrm{CH}_{3}\right), 7.21$ (dd, $J=3.7,4.7 \mathrm{~Hz}, 1 \mathrm{H}, \mathrm{ArH}), 7.49$ (m, 2H, ArH), 7.53 (dd, $J=1.09,4.68 \mathrm{~Hz} 1 \mathrm{H}, \mathrm{ArH}$, ), 7.69 (dd, $J=1.1,3.72 \mathrm{~Hz}, 1 \mathrm{H}, \mathrm{ArH}), 7.80(\mathrm{~d}, J=1.27 \mathrm{~Hz}, 1 \mathrm{H}, \mathrm{ArH})$. Anal. Cald. for $\mathrm{C}_{15} \mathrm{H}_{12} \mathrm{O}_{2} \mathrm{~S}_{2}$ (288.24): C, 62.50; H, 4.19. Found: C, 62.60; H, 4.31.

1-(3-Phenylbenzo[b]thiophen-2-yl)ethanone (8e). Colorless crystals, mp $70{ }^{\circ} \mathrm{C}$. IR (KBr): 1682 (CO) $\mathrm{cm}^{-1} ;{ }^{1} \mathrm{H}$ NMR $\left(\mathrm{CDCl}_{3}\right) \delta 2.10$ (s, 3H, $\left.\mathrm{CH}_{3}\right), 7.38(\mathrm{~m}, 3 \mathrm{H}, \mathrm{ArH}), 7.45$ (d, $\left.J=8.4 \mathrm{~Hz} 1 \mathrm{H}, \mathrm{ArH}\right)$, 7.53 (m, 3H, ArH), 7.89 (d, $J=8.26 \mathrm{~Hz}, 1 \mathrm{H}, \mathrm{ArH}) ;{ }^{13} \mathrm{C}$ NMR $\left(\mathrm{CDCl}_{3}\right): 194.8$ (CO), 142.1, 141.0; 140.9, 140.8, $134.2\left(\mathrm{C}_{\mathrm{q}}\right)$, 129.7, 128.9, 128.7, 127.5, 125.7, 124.8, $122.6(\mathrm{CH}), 29.7\left(\mathrm{CH}_{3}\right)$. Anal.. Cald. for $\mathrm{C}_{16} \mathrm{H}_{12} \mathrm{OS}$ (252.25): C, 76.17; H, 4.79. Found: C, 76.25; H, 4.85.

1-[3-(Furan-2-yl)benzo[b]thiophen-2-yl]ethanone (8f). Colorless crystals, mp $69{ }^{\circ} \mathrm{C}$. IR (KBr): 1677 (CO) cm ${ }^{-1}$; ${ }^{1} \mathrm{H}$ NMR $\left(\mathrm{CDCl}_{3}\right) \delta 2.87$ (s, 3H, $\left.\mathrm{CH}_{3}\right), 6.66$ (m, 1H), 7.48 (m, 2H, ArH), 7.68 (m, 1H,ArH) 7.89(m, 2H,ArH), 8.04(m, 1H,ArH). Anal. Cald. for $\mathrm{C}_{14} \mathrm{H}_{10} \mathrm{O}_{2} \mathrm{~S}$ (242.22):C 69.41,H 4.16;Found: C 69.45, H 4.10

1-[3-(Thiophen-2-yl)benzo[b]thiophen-2-yl-ethanone (8g). Yellow oil. ${ }^{1} \mathrm{H}$ NMR $\left(\mathrm{CDCl}_{3}\right)$ : $\delta$ 2.25 (s, 3H, $\mathrm{CH}_{3}$ ), 7.17 (d, $\left.J=3.32 \mathrm{~Hz}, 1 \mathrm{H}, \mathrm{ArH}\right), 7.23$ (m, 1H, ArH), 7.37 (m, 1H, ArH), 7.48 (m, 1H, ArH), 7.57 (d, $J=5.80 \mathrm{~Hz}, 1 \mathrm{H}, \operatorname{ArH}), 7.60$ (d, $J=8.12 \mathrm{~Hz}, 1 \mathrm{H}, \operatorname{ArH}), 7.88$ (d, $J=7.90$ $\mathrm{Hz}, 1 \mathrm{H}, \mathrm{ArH}) ;{ }^{13} \mathrm{C} \mathrm{NMR}\left(\mathrm{CDCl}_{3}\right): \delta 193.6$ (CO), 142.9, 141.2; 140.1, 134.2, $133.6\left(\mathrm{C}_{\mathrm{q}}\right), 129.3$, 
128.8, 127.6, 125.5, 125.1, 124.8, $122.6(\mathrm{CH}), 29.7\left(\mathrm{CH}_{3}\right)$. Anal. Cald. for $\mathrm{C}_{14} \mathrm{H}_{10} \mathrm{OS}_{2}$ (226.22): C, 74.32; H, 4.45. Found: C, 74.10; H, 4.52.

1-(3-Phenylbenzoselenophen-2-yl)ethanone (8h). Colorless crystals, mp $92{ }^{\circ} \mathrm{C} .{ }^{1} \mathrm{H}$ NMR $\left(\mathrm{CDCl}_{3}\right): \delta 2.01\left(\mathrm{~s}, 3 \mathrm{H}, \mathrm{CH}_{3}\right), 7.35(\mathrm{~m}, 5 \mathrm{H}, \mathrm{ArH}), 7.52(\mathrm{~m}, 2 \mathrm{H}, \mathrm{ArH}), 7.54(\mathrm{~d}, J=7.59 \mathrm{~Hz}, 1 \mathrm{H}$, $\mathrm{ArH}), 7.93$ (d, $J=7.86 \mathrm{~Hz} 1 \mathrm{H}, \mathrm{ArH}) ;{ }^{13} \mathrm{C}$ NMR $\left(\mathrm{CDCl}_{3}\right): \delta 194.8$ (CO), 145.7, 145.1; 144.2, 142.8, $136.5\left(\mathrm{C}_{q}\right), 129.5,128.8,128.0,127.4,125.7,124.9(\mathrm{CH}), 29.3\left(\mathrm{CH}_{3}\right)$. Anal. Cald. for $\mathrm{C}_{16} \mathrm{H}_{12} \mathrm{OSe}$ (299.16): C, 64.22; H, 4.04. Found: C, 64.10; H, 4.14.

Preparation of oximes 9a-g. Sodium acetate (127 mg, $1.5 \mathrm{mmol}$ ) and hydroxylamine hydrochloride (119 mg, $1.5 \mathrm{mmol}$ ) were added to a solution of ketone $\mathbf{8 a}-\mathbf{h}$ (1 mmol.) in ethanol $(10 \mathrm{~mL})$. The resulting solution was stirred under reflux for $3 \mathrm{~h}$ and was then poured into ice water. The resulting precipitate was filtered off, washed with water and dried in vacuum at $60^{\circ} \mathrm{C}$.

1-(5-Bromo-3-phenylbenzo[b]furan-2-yl)ethanone oxime (9a). Yellow solid; mp $176{ }^{\circ} \mathrm{C} .{ }^{1} \mathrm{H}$ NMR $\left(\mathrm{CDCl}_{3}\right): \delta 2.05$ (s, 3H, $\left.\mathrm{CH}_{3}\right), 7.45(\mathrm{~m}, 8 \mathrm{H}, \mathrm{ArH}), 7.57$ (s, $\left.1 \mathrm{H}, \mathrm{OH}\right)$.

1-(5-Chloro-3-phenylbenzo[b]furan-2-yl)ethanone oxime (9b). Beige solid; mp $168{ }^{\circ} \mathrm{C} .{ }^{1} \mathrm{H}$ NMR $\left(\mathrm{CDCl}_{3}\right): \delta 2.06$ (s, 3H, $\left.\mathrm{CH}_{3}\right), 7.31(\mathrm{dd}, J=2.32,8.7 \mathrm{~Hz} 1 \mathrm{H}, \mathrm{ArH}), 7.42(\mathrm{~d}, J=2.06 \mathrm{~Hz} 1 \mathrm{H}$, ArH), 7.46(m, 6H, ArH).

1-[3-(Thiophen-2-yl)benzo[b]furan-2-yl]ethanone oxime (9c). Yellow solid; mp $106{ }^{\circ} \mathrm{C} .{ }^{1} \mathrm{H}$ NMR ( $\mathrm{CDCl}_{3}$ ): $\delta 2.19$ (s, 3H, $\left.\mathrm{CH}_{3}\right), 7.17$ (dd, $\left.1 \mathrm{H}, \mathrm{ArH}\right), 7.27$ (m, 1H, ArH), 7.30 (d, $J=4.17 \mathrm{~Hz}$, 1H, ArH), 7.38 (m, 1H, ArH), 7.44 (d, $J=4.26 \mathrm{~Hz}, 1 \mathrm{H}, \operatorname{ArH}), 7.53$ (d, $J=8.20 \mathrm{~Hz}, 1 \mathrm{H}, \operatorname{ArH}$ ), 7.66 (d, $J=8.06 \mathrm{~Hz}, 1 \mathrm{H}, \mathrm{ArH}), 8.20$ (s, 1H, OH).

1-[5-Methylsulfanyl-3-(thiophen-2-yl)benzo[b]furan-2-yl]ethanone oxime (9d). Yellow solid; mp $119{ }^{\circ} \mathrm{C} .{ }^{1} \mathrm{H}$ NMR $\left(\mathrm{CDCl}_{3}\right): \delta 2.17\left(\mathrm{~s}, 3 \mathrm{H}, \mathrm{CH}_{3}\right), 2.5$ (s, 3H, $\left.\mathrm{SCH}_{3}\right), 7.16(\mathrm{~m}, 1 \mathrm{H}, \mathrm{ArH}), 7.29$ (dd, $J=1.0,3.6 \mathrm{~Hz}, 1 \mathrm{H}, \mathrm{ArH}), 7.34$ (dd, $J=1.86,8.71 \mathrm{~Hz}, 1 \mathrm{H}, \mathrm{ArH}), 7.44(\mathrm{~d}, J=8.77 \mathrm{~Hz}, 1 \mathrm{H}$, ArH), 7.49 (dd, $J=1.27,5.2 \mathrm{~Hz}, 1 \mathrm{H}, \mathrm{ArH}$ ), 7.56 (d, $J=1.69 \mathrm{~Hz} 1 \mathrm{H}, \mathrm{ArH})$.

1-(3-Phenylbenzo[b]thiophen-2-yl)ethanone oxime (9e). Beige solid; mp $168{ }^{\circ} \mathrm{C} .{ }^{1} \mathrm{H}$ NMR $\left(\mathrm{CDCl}_{3}\right): \delta 1.84\left(\mathrm{~s}, 3 \mathrm{H}, \mathrm{CH}_{3}\right), 7.31(\mathrm{~d}, J=7.68 \mathrm{~Hz}, 1 \mathrm{H}, \mathrm{ArH}), 7.41(\mathrm{~m}, 1 \mathrm{H}, \mathrm{ArH}), 7.49(\mathrm{~m}, 4 \mathrm{H}$, ArH), 7.83 (d, $J=7.85 \mathrm{~Hz}, 1 \mathrm{H}, \mathrm{ArH}), 9.09$ (s, 1H, OH).

1-[3-(Furan-2-yl)benzo[b]thiophen-2-yl]ethanone oxime (9f). Beige solid; mp $148{ }^{\circ} \mathrm{C} .{ }^{1} \mathrm{H}$ NMR (CDCl $)$ ) $\delta 1.99$ (s, 3H, $\left.\mathrm{CH}_{3}\right), 6.59(\mathrm{~m}, 1 \mathrm{H}, \mathrm{ArH}), 6.62$ (d, $\left.J=3.86 \mathrm{~Hz} 1 \mathrm{H}, \mathrm{ArH}\right), 7.39(\mathrm{~m}$, 2H, ArH), 7.60 (d, $J=1.70 \mathrm{~Hz}, 1 \mathrm{H}, \mathrm{ArH}), 7.82$ (dd, $J=1.86,8.30 \mathrm{~Hz}, 1 \mathrm{H}, \mathrm{ArH}$ ), 7.85 (dd, $J=$ 2.03, $8.03 \mathrm{~Hz} \mathrm{1H,} \mathrm{ArH),} 8.35$ (s, 1H, OH).

1-[3-(Thiophen-2-yl)benzo[b]thiophen-2-yl]ethanone oxime (9g). Yellow solid; mp $173^{\circ} \mathrm{C}$. ${ }^{1} \mathrm{H}$ NMR $\left(\mathrm{CDCl}_{3}\right): \delta 1.95$ (s, 3H, $\left.\mathrm{CH}_{3}\right), 7.14(\mathrm{dd}, J=1.16,3.59 \mathrm{~Hz}, 1 \mathrm{H}, \mathrm{ArH}), 7.18(\mathrm{~m}, 1 \mathrm{H}, \mathrm{ArH})$, 7.37 (m, 2H, ArH), 7.49 (dd, $J=1.064 .87 \mathrm{~Hz}, 1 \mathrm{H}, \mathrm{ArH}), 7.67$ (dd, $J=1.90,8.22 \mathrm{~Hz}, 1 \mathrm{H}, \mathrm{ArH}$ ), 7.81 (dd, $J=1.64,8.69 \mathrm{~Hz}, 1 \mathrm{H}, \mathrm{ArH}), 8.13$ (s, 1H, OH).

1-(3-Phenylbenzo[b]selenophen-2-yl)ethanone oxime (9h). Yellow solid; mp $172{ }^{\circ} \mathrm{C}$; ${ }^{1} \mathrm{H}$ NMR $\left(\mathrm{CDCl}_{3}\right): \delta 1.73$ (s, 3H, $\left.\mathrm{CH}_{3}\right), 7.29(\mathrm{~m}, 2 \mathrm{H}, \mathrm{ArH}), 7.36(\mathrm{~m}, 3 \mathrm{H}, \mathrm{ArH}), 7.46(\mathrm{~m}, 3 \mathrm{H}, \mathrm{ArH}), 7.67$ (s, $1 \mathrm{H}, \mathrm{OH}$ ), 7.85 (dd, $J=1.20,7.87 \mathrm{~Hz} 1 \mathrm{H}, \mathrm{ArH}$ ). 
Preparation of $\boldsymbol{O}$-(2,4-dinitrophenyl)oximes (10a-h). A solution of oxime $\mathbf{9 a}-\mathbf{h}$ (1 mmol) in dry THF (3 mL) was added dropwise to a suspension of $\mathrm{NaH}(60 \%, 26 \mathrm{mg}, 1.1 \mathrm{mmol})$ in dry THF (1mL) while keeping the temperature below $5{ }^{\circ} \mathrm{C}$. The resulting solution was stirred at room temperature for 1 hour. Then, a solution of 1-chloro-2,4-dinitrobenzene (255 mg,1.15 mmol) in dry THF ( $2 \mathrm{~mL}$ ) was added dropwise. The reaction mixture was stirred at room temperature overnight and poured into ice water. After addition of dichloromethane $(20 \mathrm{~mL})$ the two layers separated; the organic layer was washed with brine, dried over $\mathrm{Na}_{2} \mathrm{SO}_{4}$, and the solvent evaporated in vacuum. The crude product was purified by column chromatography (dichloromethane/cyclohexane 1:1).

1-(5-Bromo-3-phenylbenzo[b]furan-2-yl)ethanone $\quad \boldsymbol{O}$-(2,4-dinitrophenyl)oxime (10a). Yellow solid; mp $186{ }^{\circ} \mathrm{C} .{ }^{1} \mathrm{H}$ NMR $\left(\mathrm{CDCl}_{3}\right): \delta 2.56$ (s, 3H, $\left.\mathrm{CH}_{3}\right), 7.19(\mathrm{td}, 1 \mathrm{H}, \mathrm{ArH}), 7.53(\mathrm{~m}$, 7H, ArH), 7.62 (d, $J=9.38 \mathrm{~Hz}, 1 \mathrm{H}, \mathrm{ArH}), 8.21$ (dd, $J=2.69,9.41 \mathrm{~Hz}, 1 \mathrm{H}, \mathrm{ArH}), 8.86$ (d, $J=$ $2.54 \mathrm{~Hz} 1 \mathrm{H}, \mathrm{ArH})$.

1-(5-Chloro-3-phenylbenzo[b]furan-2-yl)ethanone $\boldsymbol{O}$-(2,4-dinitrophenyl)oxime (10b). Yellow solid; mp $191{ }^{\circ} \mathrm{C} .{ }^{1} \mathrm{H}$ NMR $\left(\mathrm{CDCl}_{3}\right): \delta 2.57$ (s, 3H, $\left.\mathrm{CH}_{3}\right), 7.21(\mathrm{~d}, J=9.42 \mathrm{~Hz}, 1 \mathrm{H}$, ArH,), 7.42 (dd, $J=2.17,8.8 \mathrm{~Hz}, 1 \mathrm{H}, \mathrm{ArH}), 7.52(\mathrm{~d}, J=2.51 \mathrm{~Hz}, 1 \mathrm{H}, \mathrm{ArH}), 7.52$ (m, 6H, ArH), 8.21 (dd, $J=2.72,9.37 \mathrm{~Hz}, 1 \mathrm{H}, \mathrm{ArH}), 8.86$ (d, $J=2.68 \mathrm{~Hz}, 1 \mathrm{H}, \mathrm{ArH}$ ).

1-[(3-Thiophen-2-yl)benzo[b]furan-2-yl]ethanone $\boldsymbol{O}$-(2,4-dinitrophenyl)oxime (10c). Yellow solid; mp $181{ }^{\circ} \mathrm{C} .{ }^{1} \mathrm{H}$ NMR $\left(\mathrm{CDCl}_{3}\right)$ : $\delta 2.66$ (s, 3H, $\left.\mathrm{CH}_{3}\right), 6.61(\mathrm{~m}, 1 \mathrm{H}, \mathrm{ArH}), 7.03(\mathrm{~d}, J=3.41 \mathrm{~Hz}$, 1H, ArH), 7.37( m, 1H, ArH), 7.47 (d, 1H, ArH), 7.57 (d, $J=8.05$ Hz, 1H, ArH), 7.65 (s, 1H, ArH), 7.94 (d, $J=8.54 \mathrm{~Hz}, 1 \mathrm{H}, \mathrm{ArH}), 7.96$ (d, $J=9.27 \mathrm{~Hz}, 1 \mathrm{H}, \mathrm{ArH}), 8.46$ (dd, $J=2.19,9.15$ $\mathrm{Hz}, 1 \mathrm{H}, \mathrm{ArH}), 8.94$ (d, $J=2.37 \mathrm{~Hz} 1 \mathrm{H}, \mathrm{ArH})$.

1-[5-Methylsulfanyl-3-(thiophen-2-yl)benzo[b]furan-2-yl]ethanone $O$-(2,4-dinitrophenyl)oxime (10d). Yellow solid; mp $184{ }^{\circ} \mathrm{C} .{ }^{1} \mathrm{H}$ NMR $\left(\mathrm{CDCl}_{3}\right): \delta 2.52$ (s, 3H, $\left.\mathrm{CH}_{3}\right), 2.59$ (s, 3H, $\left.\mathrm{SCH}_{3}\right), 7.27$ (m, 1H, ArH), 7.43 (dd, $J=18.57 \mathrm{~Hz}, 1 \mathrm{H}, \mathrm{ArH}), 7.55$ (m, 3H, ArH), 7.62(d, $J=9.35 \mathrm{~Hz}, 1 \mathrm{H}, \mathrm{ArH}$ ), 8.40 (dd, $J=2.54,9.38 \mathrm{~Hz}, 1 \mathrm{H}, \mathrm{ArH}), 8.95$ (d, $J=2.52 \mathrm{~Hz}, 1 \mathrm{H}, \mathrm{ArH}$ ).

1-(3-Phenylbenzo[b]thiophen-2-yl)ethanone $\boldsymbol{O}$-(2,4-dinitrophenyl)oxime (10e). Yellow solid; mp $179{ }^{\circ} \mathrm{C} .{ }^{1} \mathrm{H}$ NMR $\left(\mathrm{CDCl}_{3}\right): \delta 2.22$ (s, 3H, $\mathrm{CH}_{3}$ ), 7.36 (m, 1H, ArH), 7.42 (d, $J=7.32 \mathrm{~Hz} 1 \mathrm{H}$, ArH), 7.46 (m, 1H, ArH), 7.52 (m, 5H, ArH), 7.69 (d, $J=9.40$ Hz, 1H, ArH), 7.89 (d, 1H, $J=$ $8.04 \mathrm{~Hz} \mathrm{ArH}$ ), 8.40 (dd, $J=2.48,9.32 \mathrm{~Hz}, 1 \mathrm{H}, \mathrm{ArH}), 8.90$ (d, $J=2.38 \mathrm{~Hz} 1 \mathrm{H}, \mathrm{ArH})$.

1-[3-(Furan-2-yl)benzo[b]thiophen-2-yl]ethanone $\boldsymbol{O}$-(2,4-dinitrophenyl)oxime (10f). Yellow solid; mp $162{ }^{\circ} \mathrm{C} .{ }^{1} \mathrm{H}$ NMR $\left(\mathrm{CDCl}_{3}\right): \delta 2.30$ (s, 3H, $\left.\mathrm{CH}_{3}\right), 6.65$ (m, 1H, ArH), 6.72 (d, $J=3.00$ $\mathrm{Hz}, 1 \mathrm{H}, \mathrm{ArH}), 7.47$ (m, 2H, ArH), 7.64(d, $J=1.82 \mathrm{~Hz}, 1 \mathrm{H}, \mathrm{ArH}), 7.87$ (d, $J=8.35 \mathrm{~Hz}, 1 \mathrm{H}$, ArH), 7.89 (d, $J=8.42 \mathrm{~Hz}, 1 \mathrm{H}, \operatorname{ArH}), 7.99$ (d, $J=9.44 \mathrm{~Hz}, 1 \mathrm{H}, \mathrm{ArH}), 8.46$ (dd, $J=2.73,9.42$ $\mathrm{Hz}, 1 \mathrm{H}, \mathrm{ArH}), 8.92$ (d, $J=2.56 \mathrm{~Hz} 1 \mathrm{H}, \mathrm{ArH})$.

1-[3-(Thiophen-2-yl)benzo[b]thiophen-2-yl]ethanone $O$-(2,4-dinitrophenyl)oxime (10g). Yellow solid; mp $176{ }^{\circ} \mathrm{C} .{ }^{1} \mathrm{H}$ NMR $\left(\mathrm{CDCl}_{3}\right): \delta 2.30$ (s, 3H, $\left.\mathrm{CH}_{3}\right), 7.18(\mathrm{~m}, 2 \mathrm{H}, \mathrm{ArH}), 7.44(\mathrm{~m}$, 2H, ArH), 7.55 (d, $J=5.24 \mathrm{~Hz}, 1 \mathrm{H}, \operatorname{ArH}), 7.72$ (d, $J=8.97 \mathrm{~Hz}, 1 \mathrm{H}, \mathrm{ArH}), 7.80$ (d, $J=8.819 \mathrm{~Hz}$, 2H, ArH), 8.41 (dd, $J=2.49,8.98 \mathrm{~Hz}, 1 \mathrm{H}, \mathrm{ArH}), 8.92$ (d, $J=2.48 \mathrm{~Hz}, 1 \mathrm{H}, \mathrm{ArH}$ ). 
1-(3-Phenylbenzo[b]selenophen-2-yl)ethanone $\mathrm{O}$-(2,4-dinitrophenyl)oxime (10h). Yellow solid; mp $179{ }^{\circ} \mathrm{C} .{ }^{1} \mathrm{H}$ NMR $\left(\mathrm{CDCl}_{3}\right)$ : $\delta 2.11$ (s, 3H, $\left.\mathrm{CH}_{3}\right), 7.26(\mathrm{~m}, 1 \mathrm{H}, \mathrm{ArH}), 7.39$ (m, 4H, ArH), 7.82 (d, $J=9.05 \mathrm{~Hz}, 1 \mathrm{H}, \mathrm{ArH}), 7.92$ (d, $J=8.03 \mathrm{~Hz}, 1 \mathrm{H}, \mathrm{ArH}$ ), 8.47 (dd, $J=2.55,9.31 \mathrm{~Hz}, 1 \mathrm{H}$, ArH), 8.90 (d, $J=2.56 \mathrm{~Hz} 1 \mathrm{H}, \mathrm{ArH})$.

Cyclisation of $\boldsymbol{O}$-(2,4-dinitrophenyl)oximes (10a-h). A solution of $O$-(2,4-dinitrophenyl)oxime 10a-h $(1 \mathrm{mmol})$ in dioxane $(1.5 \mathrm{~mL})$ of was added dropwise to a suspension of $\mathrm{NaH}(60 \%, 240$ $\mathrm{mg}, 10 \mathrm{mmol})$ in dioxane $(1 \mathrm{~mL})$. The reaction mixture was heated under reflux until the starting material had disappeared (TLC). The cooled reaction mixture was poured into ice water and extracted with diethyl ether. The organic layer was separated, washed with brine, dried over $\mathrm{Na}_{2} \mathrm{SO}_{4}$, and evaporated in vacuum. The crude product was purified by column chromatography (eluent: dichloromethane).

10-Bromo-6-methylbenzo[b]furo[2,3-c]quinoline (1a). Yellow solid; mp $198{ }^{\circ} \mathrm{C}$. ${ }^{1} \mathrm{H}$ NMR $\left(\mathrm{CDCl}_{3}\right): \delta 3.01$ (s, 3H, $\left.\mathrm{CH}_{3}\right), 7.65$ (d, $\left.J=7.76 \mathrm{~Hz}, 1 \mathrm{H}, \mathrm{ArH}\right), 7.72$ (m, 2H, ArH), 7.74 (dd, $J=$ 1.77, $7.74 \mathrm{~Hz}, 1 \mathrm{H}, \mathrm{ArH}$ ), 8.24 (dd, $J=2.44,8.33 \mathrm{~Hz}, 1 \mathrm{H}, \mathrm{ArH}$ ), 8.43 (dd, $J=2.67,8.17 \mathrm{~Hz}, 1 \mathrm{H}$, ArH), 8.53 (d, $J=1.57 \mathrm{~Hz}, 1 \mathrm{H}, \mathrm{ArH}) ;{ }^{13} \mathrm{C}$ NMR: $\delta 154.8,146.8,143.7,138.7,138.3,125.6,123.2$, 116.8 (C ), 131.4, 129.7, 127.8, 127.0, 125.9, 123.1, $114.2(\mathrm{CH}), 19.7\left(\mathrm{CH}_{3}\right)$. Anal. Cald. for $\mathrm{C}_{16} \mathrm{H}_{10} \mathrm{BrNO}$ (312.16): C, 61.55; H, 3.23; N, 4.49. Found: C, 61.48; H, 3.19; N, 4.35.

10-Chloro-6-methylbenzo[b]furo[2,3-c]quinoline (1b). Yellow solid mp $191{ }^{\circ} \mathrm{C}$. ${ }^{1} \mathrm{H}$ NMR $\left(\mathrm{CDCl}_{3}\right): \delta 3.01$ (s, 3H, $\left.\mathrm{CH}_{3}\right), 7.59$ (m, 1H, ArH), 7.70 (d, J = $\left.7.72 \mathrm{~Hz} 1 \mathrm{H}, \mathrm{ArH}\right), 7.74$ (m, 2H, ArH), 8.21 (dd, $J=2.23,8.22 \mathrm{~Hz}, 1 \mathrm{H}, \mathrm{ArH}), 8.36$ (s, 1H, ArH), 8.45 (dd, $J=8.19 \mathrm{~Hz}, 1 \mathrm{H}, \operatorname{ArH}$ ); ${ }^{13} \mathrm{C}$ NMR: $\delta$ 166.0, 154.3, 151.0, 146.8, 144.4, 129.4, 124.9, 123.2 (C), 129.8, 128.6, 127.6, 127.0, 123.0, 122.8, $113.7(\mathrm{CH}), 22.7\left(\mathrm{CH}_{3}\right)$. Anal. Cald. for $\mathrm{C}_{16} \mathrm{H}_{10} \mathrm{ClNO}$ (267.70): C, 71.78; H, 3.76; N, 5.23. Found: C, 71.65; H, 3.80; N, 5.21.

6-Methylbenzo[b]seleno[2,3-c]quinoline (1c). Yellow solid; mp $134{ }^{\circ} \mathrm{C} .{ }^{1} \mathrm{H}$ NMR $\left(\mathrm{CDCl}_{3}\right): \delta$ 2.98 (s, 3H, $\mathrm{CH}_{3}$ ), 7.55 (m, 1H, ArH), 7.72 (m, 3H, ArH), 8.10 (d, J = 7.84 Hz, 1H, ArH), 8.23 (d, $J=7.94 \mathrm{~Hz}, 1 \mathrm{H}, \mathrm{ArH}), 8.93$ (d, $J=8.07 \mathrm{~Hz}, 2 \mathrm{H}$, ArH). Anal. Cald. for $\mathrm{C}_{16} \mathrm{H}_{11} \mathrm{NSe}$ (296.16): C, 64.87; H, 3.74; N, 4.73. Found: C, 64.78; H, 3.82; N, 4.75.

6-Methylbenzo[b]thieno[2,3-c]quinoline (1d). Yellow solid; mp $105{ }^{\circ} \mathrm{C} .{ }^{1} \mathrm{H}$ NMR $\left(\mathrm{CDCl}_{3}\right): \delta$ 3.00 (s, 3H, $\mathrm{CH}_{3}$ ), 7.66 (m, 2H, ArH), 7.74 (m, 2H, ArH), 8.06 (dd, J=1.60, $7.92 \mathrm{~Hz}, 1 \mathrm{H}, \mathrm{ArH}$ ), 8.26 (dd, $J=7.80 \mathrm{~Hz}, 1 \mathrm{H}, \mathrm{ArH}), 8.89$ (m, $2 \mathrm{H}, \mathrm{ArH}$ ). Anal. Cald. for $\mathrm{C}_{16} \mathrm{H}_{11} \mathrm{NS}$ (249.25): C, 77.09; H, 4.45; N, 5.62. Found: C, 77.20; H, 4.52; N, 5.70.

5-Methylbenzo[b]thieno[3,2-d]thieno[3,2-b]pyridine (2a). Yellow solid, m.p.147 ${ }^{\circ} \mathrm{C} .{ }^{1} \mathrm{H}$ NMR $\left(\mathrm{CDCl}_{3}\right): \delta$ 2.88(s, 3H, $\left.\mathrm{CH}_{3}\right), 7.62(\mathrm{~m}, 2 \mathrm{H}, \mathrm{ArH}), 7.71$ (d, J=5.43Hz, $\left.1 \mathrm{H}\right), 7.75$ (d, $J=5.44 \mathrm{~Hz}$, $1 \mathrm{H}$ ), 8.03 (dd, $J=3.24,7.69 \mathrm{~Hz} 1 \mathrm{H}, \mathrm{H}-7$ ), 8.44 (dd, $J=3.14,7.59 \mathrm{~Hz} 1 \mathrm{H}, \mathrm{H}-10$ ). Anal. Cald. for $\mathrm{C}_{14} \mathrm{H}_{9} \mathrm{NS}_{2}$ (255.22): C, 65.88; H, 3.55; N, 5.49. Found: C, 65.82; H, 3.52; N, 5.54.

5-Methylbenzo[b]furo[3,2-d]thieno[3,2-b]pyridine (2b). White solid; mp $98{ }^{\circ} \mathrm{C} .{ }^{1} \mathrm{H}$ NMR $\left(\mathrm{CDCl}_{3}\right): \delta 2.98$ (s, 3H, $\left.\mathrm{CH}_{3}\right), 7.52$ (m, 1H, ArH), 7.65 (m, 1H, ArH), 7.69 (d, J = 5.53 Hz, 1H, ArH), 7.74 (d, $J=8.56 \mathrm{~Hz}, 1 \mathrm{H}, \mathrm{ArH}), 7.75$ (d, $J=5.53 \mathrm{~Hz}, 1 \mathrm{H}, \mathrm{ArH}), 8.12$ (d, $J=7.55 \mathrm{~Hz}, 1 \mathrm{H}$, $\mathrm{ArH}) ;{ }^{13} \mathrm{C} \mathrm{NMR}\left(\mathrm{CDCl}_{3}\right): \delta$ 156.4, 151.1, 142.1, 141.1, 130.1, 121.6, 120.7 (C ), 129.3, 125.0, 
123.6, 122.7, $112.5(\mathrm{CH}), 19.0\left(\mathrm{CH}_{3}\right)$. Anal. Cald. for $\mathrm{C}_{14} \mathrm{H}_{9} \mathrm{NOS}$ (239.22): C, 70.28; H, 3.79; N, 5.88. Found: C, 70.50; H, 3.85; N, 5.80.

5-Methyl-9-methylsulfanylthieno[3,2-b]benzo[b]furo[3,2-d]pyridine (2c). Yellow solid, mp $157{ }^{\circ} \mathrm{C} .{ }^{1} \mathrm{H}$ NMR $\left(\mathrm{CDCl}_{3}\right): \delta 2.63\left(\mathrm{~s}, 3 \mathrm{H}, \mathrm{CH}_{3}\right), 2.94$ (s, 3H, $\left.\mathrm{CH}_{3}\right), 7.59$ (dd, $J=1.66,8.80 \mathrm{~Hz}$ 1H, ArH), 7.65(d, $J=8.81 \mathrm{~Hz}, 1 \mathrm{H}, \mathrm{ArH}), 7.66$ (d, $J=5.44 \mathrm{~Hz}, 1 \mathrm{H}, \mathrm{ArH}), 7.72$ (d, $J=5.43 \mathrm{~Hz}$ $1 \mathrm{H}, \mathrm{ArH}$ ), 7.99 (d, $J=1.65 \mathrm{~Hz}, 1 \mathrm{H}, \mathrm{ArH})$. Anal. Cald. for $\mathrm{C}_{15} \mathrm{H}_{11} \mathrm{NOS}_{2}$ (285.39): C, 63.15; $\mathrm{H}$, 3.88; N, 54.91. Found: C, 63.12; H, 3.80; N, 4.82.

5-Methylbenzo[b]thieno[3,2-d]furo[3,2-b]pyridine (3). Yellow solid, mp $93{ }^{\circ} \mathrm{C} .{ }^{1} \mathrm{H}$ NMR $\left(\mathrm{CDCl}_{3}\right): \delta 2.89\left(\mathrm{~s}, 3 \mathrm{H}, \mathrm{CH}_{3}\right), 7.12(\mathrm{~d}, J=2.11 \mathrm{~Hz}, 1 \mathrm{H}, \mathrm{ArH}), 7.61$ (m, 2H, ArH), 7.91 (d, $J=$ $2.09 \mathrm{~Hz}, 1 \mathrm{H}, \mathrm{ArH}), 7.97$ (dd, $J=1.42,8.36 \mathrm{~Hz}, 1 \mathrm{H}, \mathrm{ArH}), 8.65$ (dd, $J=2.10,9.05 \mathrm{~Hz}, 1 \mathrm{H}, \operatorname{ArH}$ );

${ }^{13} \mathrm{C}$ NMR: $\delta$ 148.6, 146.1, 142.4, 1401, 132.1, 118.2, 113.0 (C ), 128.0, 125.7, 124.9, 122.5 (CH), $30.4\left(\mathrm{CH}_{3}\right)$. Anal. Cald. for $\mathrm{C}_{14} \mathrm{H}_{9} \mathrm{NOS}$ (239.22): C, 70.28; H, 3.79; N, 5.85. Found: C, 70.40; H, 3.65; N, 5.95.

\section{References}

1. Yamaguchi, S.; Ohhira, Y.; Yamada, M.; Michitani, H. Y.; Kawase, Y. Bull. Chem. Soc. Jpn. 1990, 63, 952.

2. Deprets, S.; Kirsch, G. Eur. J. Org. Chem. 2000, 1353.

3. (a) Review: Miyaura, N., Suzuki, A. Chem. Rev. 1995, 95, 2457. (b) Gronowitz, S.; Lavitz, K. Chem. Scr. 1984, 24, 5. (c) Gronowitz, S.; Bobosik, V.; Lavitz, K. Chem. Scr. 1984, 23, 120.

4. (a) Scott, W. J.; Stille, J. K. J. Am. Chem. Soc. 1986, 108, 3033. (b) Echavarren, A. M.; Stille, J. K. J. Am. Chem. Soc. 1987, 109, 5478.

5. Uchiyama, K.; Hayashi, Y.; Narasaka, K. Synlett 1997, 445.

6. Jarkas, N.; Kirsch, G.; Seck, P. Heterocycl. Commun. 1998, 4, 227.

7. (a) Bean, F .R.; Johnson, J. R. J. Am. Chem. Soc. 1932, 54, 4415. (b) Florentin, D.; Roques, B. P.; Fournie-Zaluski, M. C. Bull. Soc. Chim. Fr. 1976, 1999. (c) Hornfeldt, A. B.; Gronowitz, S. Ark. Kemi. 1963, 21, 239. 